\title{
3D DIGITAL CADASTRE JOURNEY IN VICTORIA, AUSTRALIA
}

\author{
D. Shojaei ${ }^{\mathrm{a} *}$, H. Olfat ${ }^{\mathrm{a}}$, M. Briffa ${ }^{\mathrm{a}}$, A. Rajabifard ${ }^{\mathrm{b}}$ \\ ${ }^{a}$ Land Use Victoria, Department of Environment, Land, Water \& Planning, Level 18, 570 Bourke Street, Melbourne, \\ Australia - (Davood.Shojaei, Hamed.Olfat, Mark.Briffa)@delwp.vic.gov.au \\ ${ }^{\mathrm{b}}$ Centre for SDIs and Land Administration, Department of Infrastructure Engineering, The University of Melbourne, 3010 , \\ Australia - abbas.r@unimelb.edu.au
}

\section{Commission VI, WG VI/4}

KEY WORDS: 3D cadastre, 3D data modelling, 3D data visualisation, 3D data validation, 3D data storage, BIM, Victoria

ABSTRACT:

Land development processes today have an increasing demand to access three-dimensional (3D) spatial information. Complex land development may need to have a 3D model and require some functions which are only possible using 3D data. Accordingly, the Intergovernmental Committee on Surveying and Mapping (ICSM), as a national body in Australia provides leadership, coordination and standards for surveying, mapping and national datasets has developed the Cadastre 2034 strategy in 2014. This strategy has a vision to develop a cadastral system that enables people to readily and confidently identify the location and extent of all rights, restrictions and responsibilities related to land and real property.

In 2014, the land authority in the state of Victoria, Australia, namely Land Use Victoria (LUV), has entered the challenging area of designing and implementing a 3D digital cadastre focused on providing more efficient and effective services to the land and property industry. LUV has been following the ICSM 2034 strategy which requires developing various policies, standards, infrastructures, and tools. Over the past three years, LUV has mainly focused on investigating the technical aspect of a 3D digital cadastre.

This paper provides an overview of the $3 \mathrm{D}$ digital cadastre investigation progress in Victoria and discusses the challenges that the team faced during this journey. It also addresses the future path to develop an integrated 3D digital cadastre in Victoria.

\section{INTRODUCTION}

The limitations of traditional approach (paper/PDF-based plans) of cadastral survey and plan lodgements and registration have caused several issues to the Australian land development process. For example, the digital data produced by the surveyors is not available to other surveyors or other stakeholders of land development processes (e.g. developers, councils and referral authorities) for re-use. The survey and plan data cannot be automatically validated and examined, and $\mathrm{DCDB}^{1} \mathrm{~s}$ are not automatically updated using paper/PDF-based plans.

As a remedy to these issues, an ePlan Working Group (eWG) was formed by the ICSM which developed a national model to transfer digital cadastral survey data between the Australian surveying industry and government agencies in 2009 (Aien et al., 2012). In 2011, an ePlan Protocol was developed to map the components of the ePlan data model to LandXML data format. 2D ePlan is currently operational in New South Wales and Victoria. Other jurisdictions have also participated in the eWG and are investigating their options. The Singapore Land Authority also joined the eWG in 2013 and have adopted the ePlan Protocol for their cadastral surveying modelling and electronic lodgements (Soon, 2012).

Following the release of the ICSM's strategy on Cadastre 2034, the eWG has started to investigate the requirements for supporting 3D building subdivisions in ePlan. Cadastre 2034 Strategy has a vision to enable people to understand their rights, restrictions, and responsibilities (RRRs) related to land and property in a survey accurate and $3 \mathrm{D}$ environment. This vision leads to changes in current subdivisional processes. From the process point of view, 3D data must be available to provide accurate information of the land and property. This often leads to new method of data collection and sourcing. Having the

\footnotetext{
* Corresponding author

${ }^{1}$ Digital Cadastre Database
}

required $3 \mathrm{D}$ data, the analysis and the registration will give a better picture of RRRs (ICSM, 2015).

The emphasis is towards achieving a 3D digital cadastral system that enables the community and stakeholders such as councils, referral authorities, real estate agencies, insurance companies, developers, and architects to readily and confidently identify the location and related interests to land and property. The key element of this change is to ensure the efficiency of the cadastral system in Australian jurisdictions. This requires a strong commitment of stakeholders to improve the management and sharing of cadastral information and enhance their systems and infrastructures to enable this change (ICSM, 2015).

To facilitate this commitment in Victoria, LUV also formed a $3 \mathrm{D}$ working Party in 2014. The aim of this working party is to investigate the introduction of 3D digital data and its implications relevant to the cadastre. This working party has members from academia (Melbourne and RMIT Universities), the surveying industry (Institution of Surveyors Victoria, Consulting Surveyors Victoria, and Surveying and Spatial Sciences Institute), and State and Local Government Departments.

Prior to the establishment of the 3D Working Party, during 2012-2014 LUV sponsored an Australian Research Council (ARC) linkage project, '3D Land and Property Information Project' ${ }^{2}$ which was coordinated by CSDILA (the Centre for SDIs and Land Administration ${ }^{3}$ ) at the University of Melbourne. This project aimed to develop an innovative infrastructure which helps address the problem of modelling and managing complex 3D property RRRs in multi-level developments in our rapidly growing cities. This project delivered an improved understanding of the problems and issues associated with incorporating $3 \mathrm{D}$ property information into land

\footnotetext{
2 ARC-LINKAGE PROJECT (2012-2015) LP110200178, www.csdila.unimelb.edu.au/projects/3dwebsite

${ }^{3}$ www.csdila.unimelb.edu.au
} 
administration systems. The outcomes of the project also led to a new ARC linkage project namely '3D Property Ownership Map Base for Smart Urban Land Administration' recently awarded to CSDILA and sponsored by LUV, ICSM and the City of Melbourne.

In addition to the above-mentioned academic research projects, LUV commenced an in-house research project in 2014 to investigate various aspects of $3 \mathrm{D}$ digital cadastre. Among the legal, institutional and technical aspects defined for a 3D cadastre (Aien et al., 2012), the latter has been under investigation since the beginning of the project. 3D data modelling, visualisation, storage and validation have been studied so far. The outcomes of this investigation have been addressed in this paper. The paper firstly presents the current practice and challenges in representation of RRRs in Victoria.
The 3D digital cadastre research outcomes are discussed in Section 2. Section 3 discusses the future directions identified as part of this research. It finally concludes the paper in section 4 .

\section{3D CADASTRE IN VICTORIA - CURRENT STATUS \& RESEARCH OUTCOMES}

In Victoria, regulations allow registering overlapped RRRs in 3D under the Subdivision Act 1988 (Victorian Government, 2011). These RRRs are recorded and registered in paper or PDF based plans. They include floor plans and cross-sections to show the complexity of ownership boundaries. Figure 1 shows a sample of these plans.
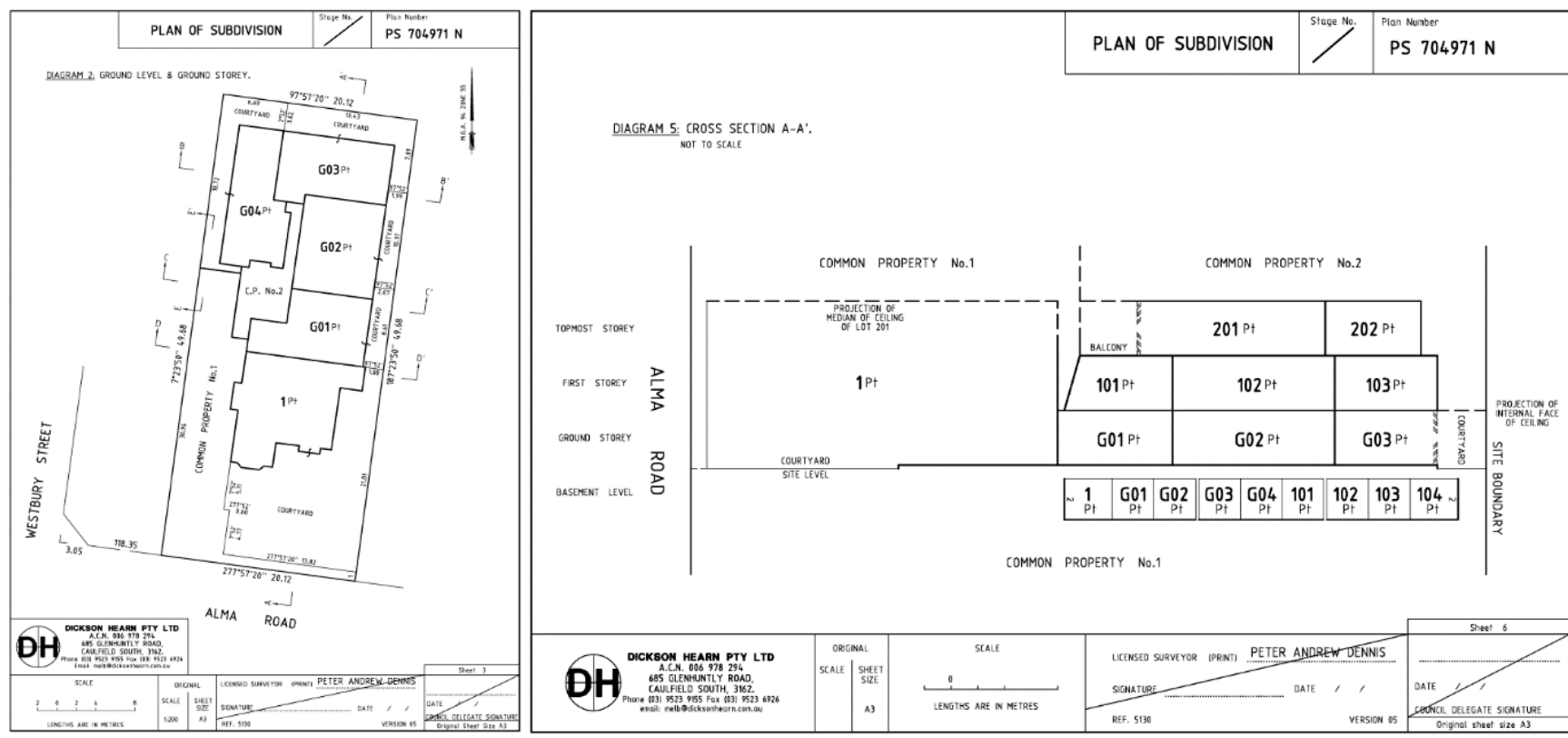

Figure 1. sample of a building subdivision plan including floor plans and cross-sections

In Victoria, these plans are called building subdivision plans. However, in other jurisdictions such as New South Wales, they are called strata plans. The building subdivision plans include cross-sections to show the extent of the ownership boundaries in a vertical axis. As shown in Figure 1, building boundaries are not dimensioned in building subdivision plans and there is no area information for parcels with building boundaries. This makes it very difficult to back capture the existing building subdivision plans and produce 3D models.
In addition to building subdivision plans, plans for underground and above ground infrastructures need to be considered for a 3D digital cadastre in Victoria. These plans are registered as Crown Allotment plans (Figure 2).

Unlike the building subdivision plans, Crown Allotment Plans (government-owned land) include dimensions to show the extent of the infrastructure. 

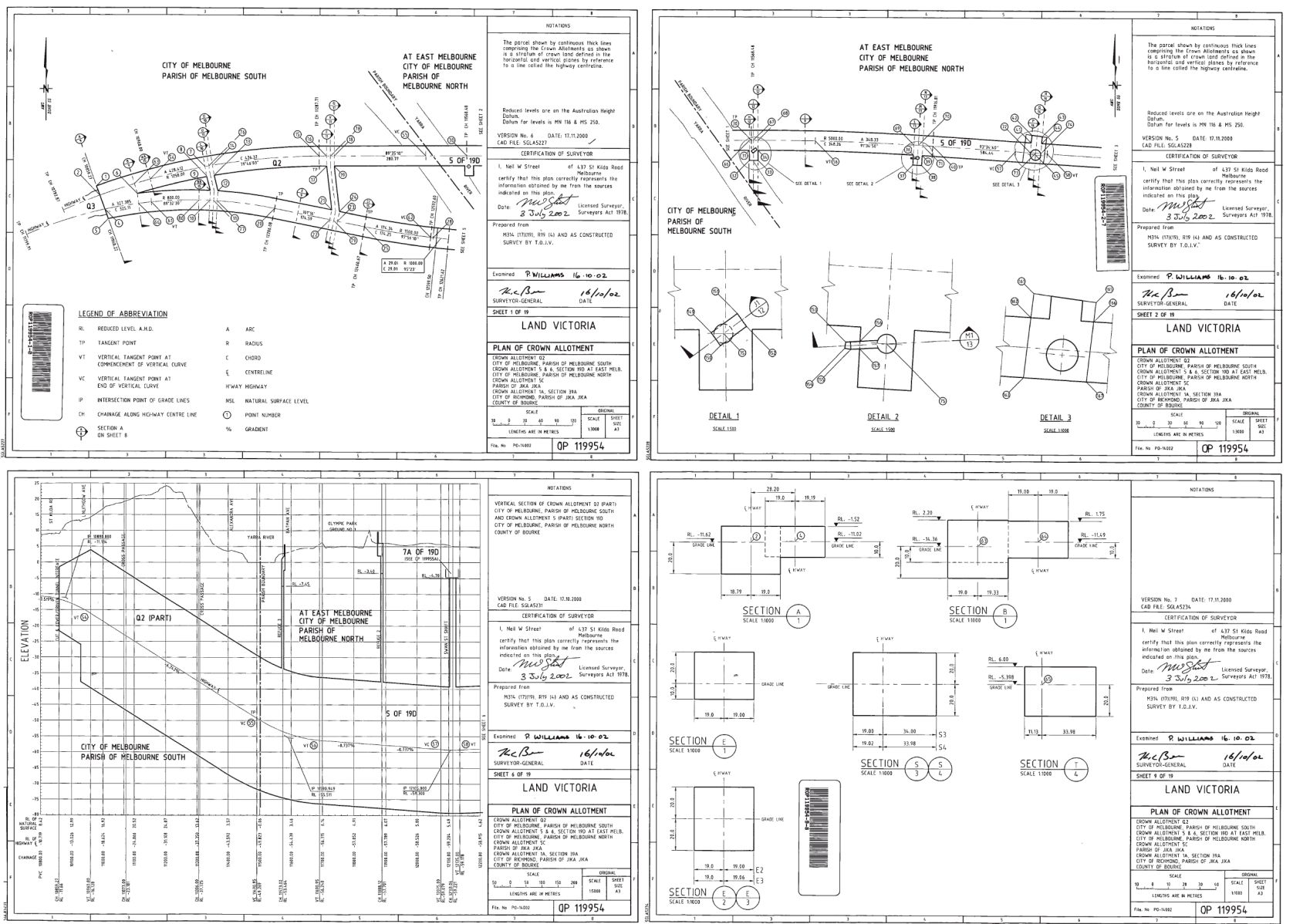

Figure 2. sample of Crown Allotment plan showing a tunnel in Melbourne

In the Victorian DCDB (Vicmap Property), multi storey building subdivisions are stored as 2D parcels. Figure 3 shows a multi storey building in the LASSI (Land and Spatial Survey Information $^{4}$ ) application. LASSI can be used to find a parcel of land or property online, or to identify the boundary of a property and those of surrounding properties. However, this method of representation cannot efficiently represent the details of overlapped ownership rights properly.

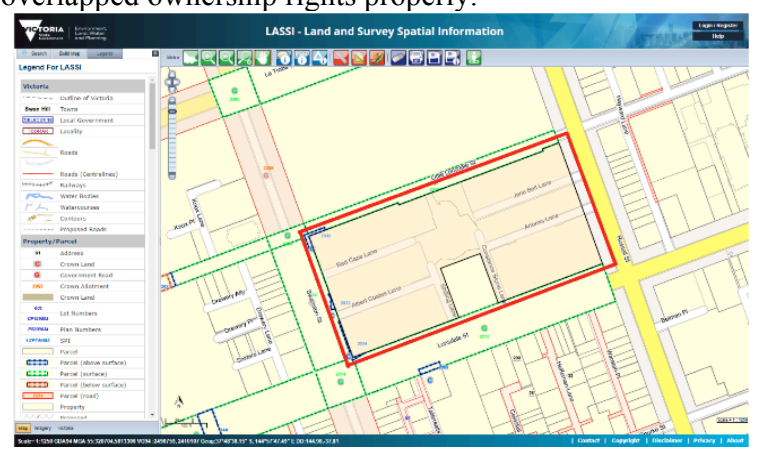

Figure 3. multi storey building shown in LASSI application

Since 2014, various technical areas have been investigated as part of the Victorian 3D Digital Cadastre. The technical aspect looks at $3 \mathrm{D}$ visualisation, validation and modelling to support building subdivision plans in Victoria. This section provides an overview of current activities.

\footnotetext{
${ }^{4}$ maps.land.vic.gov.au/lassi/
}

\subsection{D Data Visualisation}

As part of the 3D data visualisation investigation, an interactive prototype has been developed which illustrates how the legal and physical objects of a building subdivision plan can be stored, visualised and queried in a $3 \mathrm{D}$ web-based visualisation application. The aim of developing this prototype was to communicate the 3D digital cadastre concept with people involved in the land development process. WebGL technology has been utilised for implementing this prototype. The underlying data is based on Building Information Modelling (BIM) and ownership rights have been defined as IfcSpace elements in the data. Attribute data for each ownership space has been added to this 3D model and related 3D objects (e.g. lots and its carpark) have been linked for queries in the prototype. Figure 4 shows a snapshot of this prototype.

This prototype is available on the SPEAR website ${ }^{5}$. The second version of this prototype has improved the performance of the system and added several new features such as cross-section view, measurement, and support for popular internet browsers. 


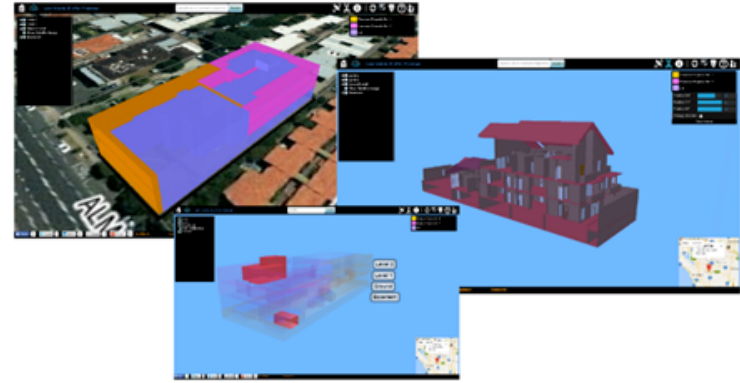

Figure 4. Land Use Victoria 3D ePlan prototype

\subsection{D Data Validation}

Data ambiguity and invalidity can cause significant expensive issues in the cadastral domain (e.g. legal disputes). Therefore, an automated data validation can significantly help to reduce the potential issues. Quality assurance has been comprehensively investigated in various domains. However, the validation of 3D cadastral data is still in its infancy and has only recently started to develop. The availability of various regular and irregular shapes for 3D cadastral objects and modern building designs has resulted in a critical need for developing validation rules to ensure data validity and quality.

This study is being undertaken in three main phases including 1) developing geometrical validation rules, 2) developing nongeometrical validation rules, 3 ) implementing an online service to validate $3 \mathrm{D}$ ePlan. Figure 5 illustrates the phases defined for $3 \mathrm{D}$ data validation investigation.

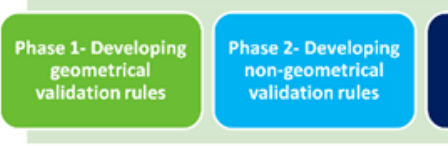

Figure 5. defined phases in 3D data validation investigation

Currently, phase one of this study is under investigation. As part of this study, four geometrical validation rules have been formalised using mathematical expressions to check the individual 3D parcels and their relationships with adjoining or neighbouring parcels. The first validation rule checks the compatibility of the subdivided parcel against the created parcels. The second rule ensures the faces forming a 3D parcel are flat. The third rule assures $3 \mathrm{D}$ objects are watertight. The fourth validation rule deals with parcel collision detection which is required for flagging unacceptable intersection of $3 \mathrm{D}$ objects.

\subsection{D Data Modelling}

In this research, the national ePlan LandXML Protocol was comprehensively investigated in three main phases to understand how it can model various building subdivision plans. In the first phase, a simple building subdivision plan was successfully modelled in LandXML and the feasible modelling approach was identified. In phase 2, a complex building subdivision was modelled and in the last phase, a building with curved surfaces was modelled in LandXML. It was concluded that buildings with flat faces could be modelled in LandXML. However, buildings with curved surfaces are approximated with small flat faces. This is due to the limitation of LandXML for 3D modelling of irregular shaped buildings (Shojaei et al., 2016).

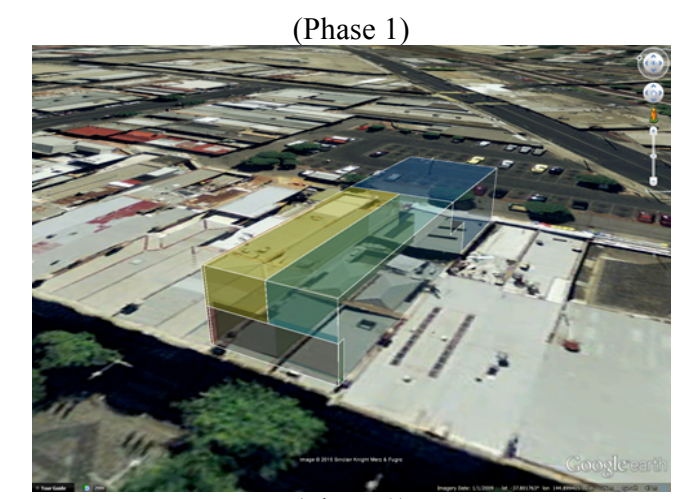

(Phase 2)

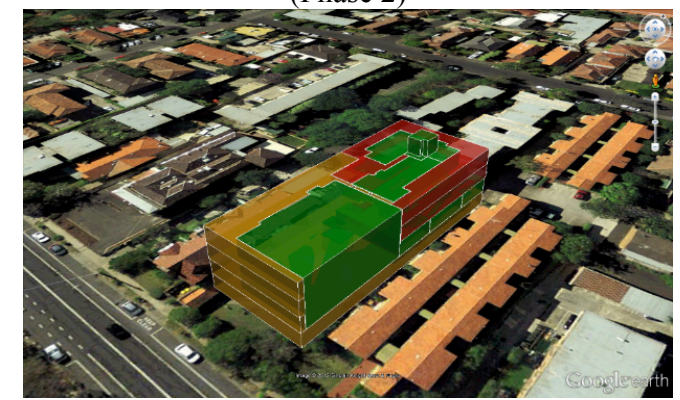

(Phase 3)
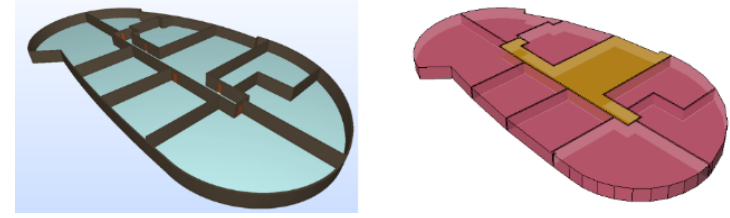

Figure 6. investigating the three phases in creating various building subdivision plans in ePlan

\subsection{Supporting Building Subdivision Plans in ePlan}

In Victoria, building subdivision plans are not currently supported in ePlan. The issue is related to complexity of overlapping ownership rights. Currently, there is no surveying software that supports building subdivision plans in ePlan, and there are limitations in software packages for preparing 3D objects and exporting them to ePlan format. A case study was defined to find an approach for preparing a building subdivision plan in ePlan. For this investigation, four different packages were tested and utilised to create a $3 \mathrm{D}$ ePlan file. Figure 7 shows the steps and the packages used in this process.

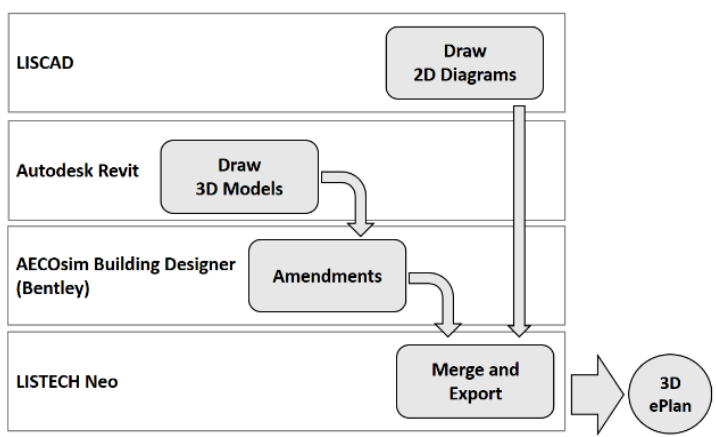

Figure 7. defined steps and utilised software for creating a 3D ePlan

In the first step, a simple building subdivision plan was selected with 12 lots, and two common property parcels. LISCAD 
package was utilised to back capture the survey plan including the land parcel and traverses in 2D.

Autodesk Revit was next used to capture the 3D model of the building. Revit is mainly used by architects to design buildings; however, it has limitations in modelling of some specific objects. For example, very narrow objects cannot be defined in Revit as a space, and, there are also limitations in defining intersected $3 \mathrm{D}$ objects. The $3 \mathrm{D}$ model was exported in IFC format $^{6}$.

To resolve the issues identified in Revit, the 3D model was imported into AECOsim Building Designer. This tool can edit 3D models and it resolved the issues identified in Revit. In AECOsim Building Designer, narrow objects can be defined and there is no problem with intersected spaces. After resolving the issues, the 3D model was exported in IFC format again.

Finally, the amended IFC file was imported into LISTECH Neo to merge that with the $2 \mathrm{D}$ captured plan in the first phase. LISTECH Neo is a tool which can read 2D and 3D models and export to several 3D formats.

However, the LandXML format supported in LISTECH Neo is not based on the ePlan protocol and therefore, the exported ePlan is not in a proper format based on the requirements of the Victorian cadastre. In addition, an ePlan plugin is required in LISTECH Neo to enable adding the required ePlan attributes to the 3D model. This topic is still being investigated.

The next section addresses the future direction of $3 \mathrm{D}$ digital cadastre implementation in Victoria.

\section{FUTURE DIRECTIONS}

Currently, various areas of 3D digital cadastre have been investigated and there is a long journey ahead. Some of the potential areas to investigate are seen in this paper, and the rest will appear from further investigation in the future.

Among the three aspects, the institutional and legal aspects of $3 \mathrm{D}$ digital cadastre require more investigation. The technical aspect has been considered during this investigation. This section brings an overview regarding the future investigations required in Victoria.

\subsection{D Data Visualisation}

New technologies are emerging which will change the traditional methods of visualising 3D objects. Augmented and virtual reality applications are becoming widespread and could be utilised for future development of visualisation applications for 3D cadastral purposes. Figure 8 is a conceptual design of an application to show apartment units based on Augmented Reality (AR) technique.

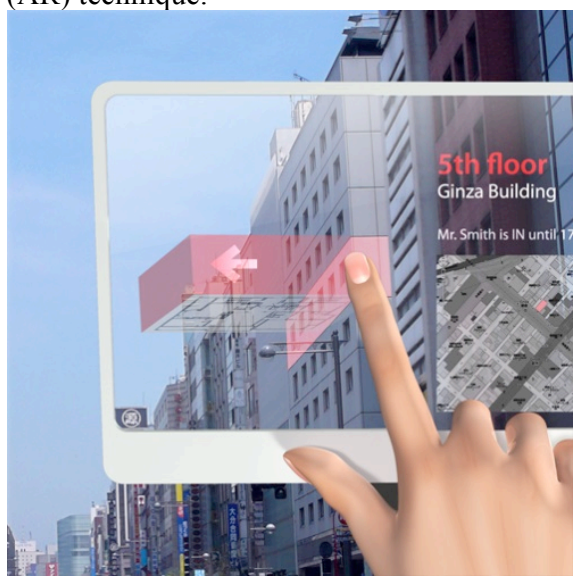

\footnotetext{
${ }^{6}$ Industry Foundation Classes
}

Figure 8. conceptual design for an augmented reality application to show $3 \mathrm{D}$ ownership rights (Courtesy of petitinvention ${ }^{7}$ )

The current prototype only shows one building and associated rights. However, like the current 2D applications (e.g. LASSI), a 3D web-based application would be useful. Therefore, a 3D visualisation application for showing ownership rights in a city scale is required.

The current prototype will be evaluated by plan examiners at LUV and surveyors to identify the issues and limitations of the prototype and extract the requirements for the third release of this prototype.

Investigation of using semantic knowledge for visualisation applications will further improve the development of efficient visualisation applications for cadastral users. Future visualisation applications should provide users with the ability to easily understand their rights, restrictions and responsibilities. As a requirement, there is a need to include a feature to select which aggregation level (ie. which part of an object) should be selected with the 'Identify Tool' in the 3D prototype. For example, by clicking on a wall of a building, the attributes of the wall may be required or the attributes of the apartment unit. Therefore, an investigation into an appropriate 'Identify Tool for the visualisation application is suggested (Shojaei, 2014).

\subsection{D Data Validation}

According to figure 5, more validation rules including geometrical and semantical rules are required to be developed in phase one and two and will need to be implemented as a validation service.

Currently, a 2D validation service including 130 rules has been implemented for ePlan to check the quality of $2 \mathrm{D}$ ePlans submitted for registration.

This requires investigating various possible data issues and developing methods to identify errors in $3 \mathrm{D}$ data. The existing $2 \mathrm{D}$ validation rules have been developed over several years by experimenting with different data issues. The future 3D validation service will evolve over several years to cover the common issues in data. In addition, these validation services can be used within the modelling packages to validate the data at the time of production.

\subsection{D Data Modelling}

LandXML has limitations in modelling irregular shaped buildings (Shojaei et al., 2016). Therefore, other alternatives such as BIM (IFC) needs to be investigated to assess its capabilities to support 3D legal objects (Shojaei, et al., 2015, Olfat et al., 2017, Atazadeh et al., 2017). In addition, various building scenarios must be tested in LandXML to assess the limitations of data modelling for building subdivisions.

Further investigation is also required to study the modelling of infrastructures above and below the ground as shown in Figure 2. The complexity in these cases may require several validation rules.

\subsection{D Data Storage}

Currently, registered 2D ePlans are stored as a flat file in servers. This method of storage causes some challenges such as lack of data integrity and consolidation, performance drawbacks in the future and limitations in spatial and attribute queries. During this investigation, several databases were assessed and Oracle has been identified as the most suitable, based on its high performance in storing, retrieving and analysing spatial data.

${ }^{7}$ petitinvention.wordpress.com/2009/09/04/red-dot-design-conceptaward-2009/ 
3D data can be stored in a database in two main approaches, namely the geometry and topology-based structure database. The topology-based data structure does not store redundant objects. However, geometry-based structure is more practical (Mutiarasari et al., 2014).

The next step is to investigate the best approaches for storing $3 \mathrm{D}$ legal objects within a database for cadastral purposes.

\subsection{Support to Building Subdivision Plans in ePlan}

As discussed in Section 2.4, various applications were used to export a building subdivision plan in ePlan. However, an application is required to include all the required features for exporting 3D ePlans. There have been some discussions with several software vendors in Australia to develop this application for creating building subdivision plans. In parallel, more building scenarios should be tested to investigate the challenges in preparing building subdivision plans.

\subsection{Legal Aspect}

The current Act, the Subdivision ACT 1988, allows registering of overlapped RRRs (building subdivisions) in Victoria and they are registered in an analogue (paper and PDF) approach. However, the registered data cannot be used for creating 3D models of RRRs in the future. Therefore, legislation needs to change to encourage architects, and surveyors to provide their 3D legal models when they submit their building subdivision plans. Having these 3D legal models will facilitate implementing a 3D digital cadastre in the future. In city planning, Victorian regulations require a $3 \mathrm{D}$ model for the development over 25,000 square metres ${ }^{8}$. This shows that the government is aware of the importance of $3 \mathrm{D}$ data created in the land development process. However, any change in the legislation takes time to be implemented.

There are several other topics and questions in the legal aspect that require investigation. These topics and questions include:

- What would be the legal point of truth for registration of ownership rights? 3D model or 2D plans (PDF/TIFF)?

- What should be incorporated into the contract of sale as part of marketing and transferring ownership?

- Can all stakeholders and courts use 3D legal models for their decision-making process?

- Do 3D models intend to replace or supplement 2D plans?

- What are the required changes to the existing regulations to move to $3 \mathrm{D}$ ?

- What are the main industry bodies for driving this change and to facilitate its implementation?

- How should 3D legal disputes be handled?

These questions and topics require various research activities. As this study has concentrated on the Victorian position, a comprehensive research for other jurisdictions would be required.

\subsection{Institutional Aspect}

Understanding the institutional issues in the context of implementing a 3D digital cadastre necessitates the development of strategies to support the change in processes (Ho, 2014). The institutional aspect looks at what processes and roles should change to facilitate the implementation.

\footnotetext{
${ }^{8}$ ww.planning.vic.gov.au/_data/assets/pdf_file/0022/9670/3D-Advicenote-v2.pdf
}

An institutional framework should be developed to cover the range of topics to support the implementation of a $3 \mathrm{D}$ digital cadastre. This requires an understanding of the current institutional environment and the relevant processes specifically in Victoria. Ho and Rajabifard (2012) proposed the Institutional Analysis and Development (IAD) Framework as an appropriate approach to examine the informal rules that govern land and property information transactions, and to focus the analysis at the operational level. According to their research, the future steps could be applying the theoretical approach in the empirical institutional operations of land and property registration to implement the institutional changes which facilitate the implementation of a 3D digital cadastre.

\subsection{Road Map}

Goal 4 of ICSM Cadastre 2034 Strategy defines the future cadastre is "a digital cadastre that is 3-dimensional, dynamic and survey accurate". This goal needs a detailed road map to show the future steps and milestones towards the implementation of a 3D digital cadastre. In addition, the institutional, technical and legal aspects of a 3D digital cadastre should link together to clarify the connection and relationships of the interests of the property industry, to build a comprehensive framework for implementation.

This road map should also address the required developments with specified timeframes. The roadmap should also nominate the champions to lead the roadmap and define the milestones. Kalantari and Rajabifard (2014) proposed three milestones for a $3 \mathrm{D}$ cadastre implementation. These milestones include, defining the scope of the 3D cadastre to include only high-rise and complex developments, identifying the champions to lead the project, and finally, considering the technical requirements in accordance with the needs of the stakeholders.

\section{CONCLUSION}

The 3D digital cadastre project in Victoria aims to develop an integrated system to be used by various users such as the public, developers, councils, lawyers, land administrators, owners corporation managers and real estate agents. This project continues to research and develop the required infrastructure of a 3D digital cadastre in Victoria, which is digital, spatially ccurate and three-dimensional. Various areas in the technical aspect of the 3D digital cadastre have been investigated and developed. However, institutional and legal aspects need further investigation.

As compared to other jurisdictions, LUV has made considerable progress in the $3 \mathrm{D}$ digital cadastre domain, due to its continuous collaboration with the academia, in particular the University of Melbourne. LUV has been an industry partner of the recent Australian Research Centre (ARC) Linkage projects coordinated by the University of Melbourne including '3D Land and Property Information' and '3D Property Ownership Map Base for Smart Urban Land Administration'.

In determining the future direction, various research areas were addressed. These research areas can be defined as research topics for research bodies or students to collaborate with the industry to develop and build the system. However, development of a road map should be considered as the first priority towards implementing a 3D digital cadastre.

\section{ACKNOWLEDGEMENTS}

The authors would like to thank the ICSM ePlan Working Group, LUV staff and the Centre for Spatial Data Infrastructures and Land Administration at the University of Melbourne for sharing their thoughts and contributing to this 
paper. This paper is based on the view of the authors and may not represent the view of LUV.

\section{REFERENCES}

Aien, A., Kalantari, M., Rajabifard, A., Williamson, I., Shojaei, D., 2012. Developing and testing a 3D cadastral data model: a case study in Australia. ISPRS Annals of the Photogrammetry, Remote Sensing and Spatial Information: XXII ISPRS Congress, 1-6.

Atazadeh, B., Kalantari, M., Rajabifard, A., Ho, S., 2017. Modelling building ownership boundaries within BIM environment: A case study in Victoria, Australia, Computers, Environment and Urban Systems, Volume 61, Part A, January 2017, Pages 24-38.

Ho, S., and Rajabifard, A., 2012. Delivering 3D Land and Property Management: A Consideration of Institutional Challenges in an Australian Context, 3rd International Workshop on 3D Cadastres, 2012, Shenzhen, pp. 219-242.

Ho, S., 2014. Towards 3D-enabled urban land administration: invisible constraints and strategic choices, $\mathrm{PhD}$ Thesis, Infrastructure Engineering Department, the University of Melbourne.

ICSM 2015. Cadastre 2034 Strategy, Powering Land and Property, http://www.icsm.gov.au/cadastral/Cadastre2034.pdf

Kalantari, M. and Rajabifard, A., 2014. A Roadmap to Accomplish 3D Cadastres 4th International Workshop on 3D Cadastres 9-11 November 2014, Dubai, United Arab Emirates.

Mutiarasari, W., Aditya, T., and Waljiyanto, W., 2014. Development of Structure-based Topology of 3D Spatial Databases for Storing and Querying 3D Cadastre Cases, FIG Congress 2014 Engaging the Challenges - Enhancing the Relevance Kuala Lumpur, Malaysia 16 - 21 June 2014.

Olfat, H., Shojaei, D., Briffa, M., and Rajabifard, A., 2017. The Current Status and Ongoing Investigations of 2D and 3D Digital Cadastre (ePlan) in Victoria, Australia, 10th International Symposium on Digital Earth \& Locate17, 3-6 April 2017, Sydney, Australia.

Shojaei, D., 2014. 3D Cadastral Visualisation: Understanding Users' Requirements, PhD Thesis, Infrastructure Engineering Department, the University of Melbourne.

Shojaei, D., Rajabifard, D., Kalantari, M., Bishop, I, Aien, A., 2015. Design and development of a web-based 3D cadastral visualisation prototype, International Journal of Digital Earth, 8 (7), 538-557.

Shojaei, D., Olfat, H., Rajabifard, A., Darvill, A., Briffa, M., 2016. Assessment of the Australian digital cadastre protocol (ePlan) in terms of supporting 3D building subdivisions, Land Use Policy Journal, Volume 56, November 2016, Pages 112124.

Soon, K. H., 2012. A Conceptual Framework of Representing Semantics for 3D Cadastre in Singapore 3rd International Workshop on 3D Cadastres: Developments and Practices Shenzhen, China.

Victorian Government, 2011. Subdivision Act 1988, http://www.legislation.vic.gov.au/Domino/Web_Notes/LDMS/
LTObject Store/LTObjSt5.nsf/DDE300B846EED9C7CA25761 6000A3571/4DE5957BD473E397CA25781B00180F22/\$FILE/ 88-53aa065\%20authorised.pdf, (19 Apr. 17). 